\title{
Sensor Fault Detection and Isolation Filter for Polytopic LPV Systems: A Winding Machine Application
}

Mickael Rodrigues ${ }^{\mathrm{a}}$, Mariem Sahnoun ${ }^{\mathrm{a}}$, Didier Theilliol ${ }^{\mathrm{b}}$, Jean-Christophe Ponsart ${ }^{\mathrm{b}}$

${ }^{a}$ Laboratoire d'Automatique et Génie des Procédés, Université de Lyon, Lyon, F-69003, France; Université Lyon 1,CNRS UMR 500\%, Villeurbanne, F-69622, France (e-mail: mickael.rodrigues@univ-lyon1.fr,sahnoun@lagep.univ-lyon1.fr).

${ }^{b}$ Centre de Recherche en Automatique de Nancy, Université de Lorraine, CNRS UMR 7039, France (e-mail \{jean-christophe.ponsart,didier.theilliol\}@univ-lorraine.fr).

\section{Abstract}

In this paper, a fault diagnosis method is developed for a particular class of nonlinear systems described by a polytopic Linear Parameter Varying (LPV) formulation. The main contribution consists in the synthesis of an accurate Fault Detection and Isolation (FDI) filter and also a sensor fault magnitude estimation with a quality factor. This quality factor of the filter underlines if the fault estimation can be used or not. Stability conditions of the polytopic LPV filter are studied by ensuring poly-quadratic stability with Linear Matrix Inequality (LMI) representation. The effectiveness of this global FDI scheme through LPV modelization, filter design and stability analysis, is illustrated on a real winding machine under multiple sensor faults. Keywords: Polytopic LPV system, observer, fault diagnosis, sensor, model-based, LMI, winding machine 


\section{Introduction}

Complex automated industrial systems are vulnerable to faults in instrumentation due to the fact that sensors and actuators can be deteriorated. The unexpected sensor or actuator faults may cause unacceptable deterioration of continuous operations and leads to dangerous situations for a human operator (Isermann and Balle, 1997),(Rodrigues et al., 2007). Fault Detection and Isolation (FDI) refers to the task of inferring the occurrence of faults in a process (Harris et al., 1999) and finding their root causes with various knowledge-based system strategies: quantitative models (Venkatasubramanian et al., 2003a) and qualitative models (Venkatasubramanian et al., 2003b). Model-based FDI techniques are considered to guarantee safety in industrial Process Control (Knittel et al., 2003). Several books are dedicated to these topics such as (Gertler, 1998; Chen and Patton, 1999; Chiang et al., 2001) or more recently (Ding, 2008).

However, FDI for nonlinear systems remains a challenge (Bokor and Szabo, 2009) due to the fact that a wide operating range is considered for such systems and a single LTI model could not be used for all considered operating points. Another way to deal with FDI for nonlinear systems is to study LPV systems (Poussot-Vassal et al., 2008). FDI for regular LPV systems has been considered for example in (Bokor and Balas, 2004; Zolghadri et al., 2008; Armeni et al., 2009) and FDI for descriptor LPV systems in (Hamdi et al., 2012).

Winding systems, web handling systems or roll-to-roll systems are nonlinear processes which consider a large operating range. Control and Fault Diagnosis for such systems remain a challenge for both economical point of 
view and research. In the paper of (Xiao et al., 2011), the authors have developed a Fault Diagnosis and Fault Tolerant Control strategy for a winding system. However, their theory is developed for LTI systems and they can only consider a single operating point. In the paper of (Claveau et al., 2008; Kuhm et al., 2012), the authors have synthesized and compared a LTI controller versus a LPV controller. They underline that a LTI controller can be considered for only one operating point and not for the entire operating range. In (Gassmann and Knittel, 2011), the authors have considered a winding system which has been modeled as a LPV system, and they have synthesized a LPV controller for a winding system but only a single LTI observer for estimating a parameter of the system under some assumptions. In (Benlatreche et al., 2008), the authors have developed decentralized controllers, Proportional Integral controllers by state feedback. The method consists in designing state-feedback control with the use of BMI optimization for large-scale systems such as a winding machine.

By taking into account the modelization of a winding system into a LPV form as in (Gassmann and Knittel, 2011), the authors in (Theilliol et al., 2008) have developed a FDI technique so as to generate insensitive residuals to fault by specific decoupling methods. The authors have established a sensor fault diagnosis method for nonlinear system and have developed stability conditions of an accurate Unknown Input Observer (UIO) that performs fault detection and isolation. However, no fault estimation has been provided with such method. In (Ponsart et al., 2010), the authors have developed a method with an accurate filter which was able to both isolate and estimate sensor faults. However, the stability conditions were not studied. In (Rodrigues 
et al., 2008), stability conditions of a polytopic LPV filter which allows an efficient actuator fault detection, isolation and estimation in multi-models framework was presented.

Using the latest consideration, this paper contributes to extend and to develop a sensor fault detection, isolation and estimation for nonlinear system based on discrete time LPV model. The objectives of this paper are to design a global FDI scheme for estimating a sensor fault magnitude over a wide operating range applied to a winding machine.

Thus, based on a polytopic LPV representation, this paper addresses an original contribution that could allow not only to detect, isolate and estimate multiple sensor faults but also to provide an additional indicator of the efficiency of the filter. This indicator is defined as a quality factor: close to zero if the filter decouples the appropriate faults or different from zero otherwise. The stability conditions of each polytopic LPV filter are studied through poly-quadratic stability. The polytopic LPV filter performances are tested on a real winding process. This process is well-known in the industry where identification model for control or FDI is still an open research field as in (Knittel et al., 2003; Laroche and Knittel, 2005; Ponsart et al., 2010).

The paper is organized as follows: in section 2, the FDI problem for polytopic LPV system is stated. Section 3 defines the design of the sensor fault diagnosis module under LPV representation. The stability study conditions are addressed by using Linear Matrix Inequality (LMI). Section 4 illustrates the developed method applied to a winding machine. Finally, section 5 is devoted to conclusions. 


\section{Problem Statement}

Consider the following class of affine LPV discrete systems with sensor faults:

$$
\left\{\begin{array}{l}
x_{k+1}=\widetilde{A}\left(\theta_{k}\right) x_{k}+\widetilde{B}\left(\theta_{k}\right) u_{k} \\
y_{k}=C x_{k}+F f_{k}
\end{array}\right.
$$

$x_{k} \in \mathbb{R}^{n}$ is the state vector, $u_{k} \in \mathbb{R}^{p}$ is the control input and $y_{k} \in \mathbb{R}^{m}$ represents the measured output vector. $F \in \mathbb{R}^{m \times q}$ represents the sensor fault distribution matrix and $f_{k} \in \mathbb{R}^{q}$ is the fault vector where $q<m$. The presence of such faults may lead to performance deterioration, instability of the system or the loss of the process.

The matrix $F$, which represents the sensor fault distribution matrix, is often (but not always) defined as a matrix where each one of its column are standard (unit) vector:

$$
F_{i}=[0 \ldots 1 \ldots 0]^{T}
$$

which represents the i-th sensor fault. It is a generalization of the sensor fault representation.

$\widetilde{A}(\cdot), \widetilde{B}(\cdot)$ are functions which depend affinely on the time-varying parameter vector $\theta_{k} \in \mathbb{R}^{l}$. It is also assumed that this time-varying parameter vector $\theta_{k}$ is fault-free, bounded and lies into a hypercube (Anstett et al., 2009), (Bruzelius, 2004) such that $\forall j \in[1, \ldots, l]$ :

$$
\theta_{k} \in \Gamma=\left\{\theta_{k} \mid \underline{\theta}_{k}^{j} \leq \theta_{k}^{j} \leq \bar{\theta}_{k}^{j}\right\}, \quad \forall k \geq 0
$$

The matrices $\widetilde{A}\left(\theta_{k}\right), \widetilde{B}\left(\theta_{k}\right)$ of the LPV system (1) with the affine param- 
eter dependence (3) are represented such that:

$$
\widetilde{A}\left(\theta_{k}\right)=\widetilde{A}_{0}+\sum_{j=1}^{l} \theta_{k}^{j} \widetilde{A}_{j}, \quad \widetilde{B}\left(\theta_{k}\right)=\widetilde{B}_{0}+\sum_{j=1}^{l} \theta_{k}^{j} \widetilde{B}_{j} \quad \forall \theta_{k} \in \Gamma
$$

Based on (4), the system (1) can be transformed into a convex interpolation of the $\mathrm{j}$ vertices of $\Gamma$ where the vertices of the polytope are defined such that (Rodrigues et al., 2007): $S_{j}=\left[A_{j}, B_{j}, C, F\right], \forall j \in[1, \ldots, N]$ where $N=2^{l}$. The polytopic coordinates are denoted $\rho(\theta(k))$ and vary within the convex set $\Lambda$ :

$$
\Lambda=\left\{\rho\left(\theta_{k}\right) \in \mathbb{R}^{N}, \rho\left(\theta_{k}\right)=\left[\rho_{1}\left(\theta_{k}\right), \ldots, \rho_{N}\left(\theta_{k}\right)\right]^{T}, \rho_{j}\left(\theta_{k}\right) \geq 0, \sum_{j=1}^{N} \rho_{j}\left(\theta_{k}\right)=1\right\}
$$

Consequently, the polytopic LPV system under sensor faults consideration is given by:

$$
\left\{\begin{array}{l}
x_{k+1}=\sum_{j=1}^{N} \rho_{j}\left(\theta_{k}\right)\left(A_{j} x_{k}+B_{j} u_{k}\right) \\
y_{k}=C x_{k}+F f_{k}
\end{array}\right.
$$

where $A_{j} \in \mathbb{R}^{n \times n}, B_{j} \in \mathbb{R}^{n \times p}, C \in \mathbb{R}^{m \times n}$ and $F \in \mathbb{R}^{m \times q}$ are time invariant matrices defined for the $j^{\text {th }}$ model. In (Rodrigues et al., 2008), the authors have considered actuator faults and, in this paper, sensor faults are considered. First of all, a preliminary work consists in rewriting the system (5). Based on (Park et al., 1994), in the presence of sensor faults, a system (5) can be rewritten as a system affected by a pseudo-actuator fault. Assume that the dynamic of the sensor fault is described as:

$$
f_{k+1}=\delta f_{k}+\bar{f}_{k}
$$


where $\delta \in \mathbb{R}^{q \times q}$ defined by $\delta=\operatorname{diag}\left(\delta_{1}, \ldots, \delta_{q}\right)$ and $\bar{f}_{k}$ is the sensor error input. Note that $\delta$ represents the additional degree-of-freedom in estimator design (See (Park et al., 1994)) and should be fixed between ]0, ., 1]. So, according to (5) and (6), a new fault polytopic LPV system representation is given by:

$$
\left\{\begin{aligned}
\bar{x}_{k+1} & =\sum_{j=1}^{N} \rho_{j}\left(\theta_{k}\right)\left(\bar{A}_{j} \bar{x}_{k}+\bar{B}_{j} u_{k}+\bar{F} \bar{f}_{k}\right) \\
y_{k} & =\bar{C} \bar{x}_{k}
\end{aligned}\right.
$$

with $\bar{x}_{k}=\left[\begin{array}{c}x_{k} \\ f_{k}\end{array}\right], \bar{A}_{j}=\left[\begin{array}{cc}A_{j} & \underline{0} \\ \underline{0} & \delta\end{array}\right], \bar{B}_{j}=\left[\begin{array}{l}B_{j} \\ \underline{0}\end{array}\right], \bar{F}=\left[\begin{array}{l}\underline{0} \\ I\end{array}\right]$ and, $\bar{C}=$ $\left[\begin{array}{lll}C & F\end{array}\right]$ where $\underline{0}$ means the zero matrix and $I$ the identity matrix of appropriate dimensions.

Note: This representation of sensor fault with pseudo-actuator fault is relevant to deal with Unknown Input methods developed in classical FDI framework.

The main goal in Fault Detection and Isolation (FDI) is to design a filter which is able to determine if a fault has occurred or not in the system. For this simple question, it is well-known that synthesizing a residual, which is the difference between the outputs of the system and the estimated outputs from the filter, can give an answer to this question. So, to this end, the designers can synthesize the following polytopic LPV Luenberger filter under the classical assumption that the pairs $\left(\bar{C}, \bar{A}_{j}\right)$ are observable (Chen and Patton, 1999): 


$$
\left\{\begin{array}{l}
\hat{\bar{x}}_{k+1}=\sum_{j=1}^{N} \rho_{j}\left(\theta_{k}\right)\left(\bar{A}_{j} \hat{\bar{x}}_{k}+\bar{B}_{j} u_{k}+\bar{K}_{j}\left(y_{k}-\bar{C} \hat{\bar{x}}_{k}\right)\right) \\
\hat{y}_{k}=\bar{C} \hat{\bar{x}}_{k}
\end{array}\right.
$$

or also with notation $(\cdot)\left(\rho_{k}\right)=\sum_{j=1}^{N} \rho_{j}\left(\theta_{k}\right)\left(\cdot{ }_{j}\right)$ such as:

$$
\left\{\begin{array}{l}
\left.\hat{\bar{x}}_{k+1}=\bar{A}\left(\rho_{k}\right) \hat{\bar{x}}_{k}+\bar{B}\left(\rho_{k}\right) u_{k}+\bar{K}\left(\rho_{k}\right)\left(y_{k}-\bar{C} \hat{\bar{x}}_{k}\right)\right) \\
\hat{y}_{k}=\bar{C} \hat{\bar{x}}_{k}
\end{array}\right.
$$

According to (7) and (9), the estimation error $e_{k}=\bar{x}_{k}-\hat{\bar{x}}_{k}$ and the residual $r_{k}=\bar{y}_{k}-\hat{\bar{y}}_{k}$ are given by the following relations:

$$
\left\{\begin{array}{l}
e_{k+1}=\left(\bar{A}\left(\rho_{k}\right)-\bar{K}\left(\rho_{k}\right) \bar{C}\right) e_{k}+\bar{F} \bar{f}_{k} \\
r_{k}=\bar{C} e_{k}
\end{array}\right.
$$

Usually, at this point, the goal consists in stabilizing the matrix $\left(\bar{A}\left(\rho_{k}\right)-\right.$ $\left.\bar{K}\left(\rho_{k}\right) \bar{C}\right)$ through a choice of the gain $\bar{K}\left(\rho_{k}\right)$ such that the matrix $\left(\bar{A}\left(\rho_{k}\right)-\right.$ $\left.\bar{K}\left(\rho_{k}\right) \bar{C}\right)$ is Hurwitz, i.e. its eigenvalues remain stable. So, in fault-free case (i.e. $\bar{f}_{k}=0$ ), the estimation error $e_{k}$ and the residual $r_{k}$ tend to zero. This indicates that no fault has occurred on the system. Moreover, if a fault occurs $\left(\bar{f}_{k} \neq 0\right)$ then the estimation error $e_{k}$ and the residual $r_{k}$, become different from zero and indicate that a fault has occurred.

However, this LPV Luenberger filter can not be used if there is more than one fault due to the fact that the residual $r_{k}$ would be corrupted by different information through the fault vector $\bar{f}_{k}$, i.e. the filter would be sensitive to all sensor faults: fault isolation is not possible and obviously the sensor fault estimation either. For multiple sensor faults, a classical technic consists 
in designing a LPV Luenberger filter (Chen and Patton, 1999) for only one sensor and to interpret the different residuals based on a bank of dedicated filters. This method allows to detect and isolate a fault. However, there is no possibility with such a filter to estimate the magnitude of multiple sensor faults.

In order to provide an efficient fault detection and isolation, the synthesis of a residual decoupled from sensor fault is provided by a polytopic LPV filter. This one will be presented in the next section under the following required assumptions for the existence of the polytopic LPV filter:

- The pairs $\left(\bar{C}, \bar{A}_{j}\right)$ are observable, $\forall i \in[1, \ldots, N]$.

- The number of measurements is greater than the number of faults i.e $q<m$ (for fault isolation purposes).

- The fault distribution matrix is full column rank i.e equal to $q$.

It is also assumed that the maximum value of fault detectability indexes is equal to one (Keller, 1999). It should be noted that the synthesis of a single filter for all sensor faults is not possible to achieve a fault detection, isolation and estimation task. Consequently, as presented in the next section, a bank of filters will also be designed.

\section{Sensor Fault detection, isolation and estimation with a quality factor}

\subsection{Polytopic LPV filter Design}

A conventional fault detection algorithm (Chen and Patton, 1999) is able to detect a fault by monitoring a residual, noted $r_{k}$, classically defined as a 
system-model difference output. In order to determine also the fault magnitude estimation and to estimate the state space vector, the authors in (Rodrigues et al., 2008) have proposed to design a filter such that the residual vector $r_{k}$ is split into two sub-vectors: one sub-vector is made sensitive to faults and the other sub-vector is made insensitive. The goal to split the residual into two-subvectors is directly linked with the fact that the main objective is to detect, isolate and estimate the fault with the sensitive part of the residual which is equivalent to synthesize the filter gain matrix $\bar{K}\left(\rho_{k}\right)$ from filter (9) such as

$$
\left(\bar{A}\left(\rho_{k}\right)-\bar{K}\left(\rho_{k}\right) \bar{C}\right) \bar{F}=0
$$

in a specific way. It can be noticed that the insensitive residual sub-vector is designed in order to provide an additional information named quality factor, considered for fault isolation as presented in paragraph (3.3). It is also explained how such decoupling is obtained.

If the equation (11) is satisfied and if the number of faults is strictly lower than the number of outputs for fault isolation purposes i.e. $\operatorname{rank}(\bar{C} \bar{F})=q<$ $m$, a solution to (11) was proposed for LTI systems by (Keller, 1999) which parameterized the gain $\bar{K}\left(\rho_{k}\right)$ such that:

$$
\bar{K}\left(\rho_{k}\right)=K^{A}\left(\rho_{k}\right)+K^{C}\left(\rho_{k}\right)
$$

where :

- $K^{A}\left(\rho_{k}\right)$ is defined by:

$$
K^{A}\left(\rho_{k}\right)=\omega\left(\rho_{k}\right) \Pi
$$


with $\omega\left(\rho_{k}\right)=\bar{A}\left(\rho_{k}\right) \bar{F}$ and $\Pi=(\bar{C} \bar{F})^{+}$where $(\cdot)^{+}$denotes the pseudoinverse of the matrix $(\cdot)$ throughout the paper.

- $K^{C}\left(\rho_{k}\right)$ is defined by:

$$
K^{C}\left(\rho_{k}\right)=K\left(\rho_{k}\right) \Psi
$$

with $\Psi=\beta\left(I_{m}-(\bar{C} \bar{F})(\bar{C} \bar{F})^{+}\right), \beta \in \mathbb{R}^{(m-q) \times m}$ is an arbitrary constant matrix defined so that matrix $\Psi$ is of full row rank and $K\left(\rho_{k}\right)$ is a gain to be synthesized.

Based on (13) and (14), the previous filter defined in (9) can be rewritten as a Polytopic LPV Filter defined as follows:

$$
\left\{\begin{array}{l}
\hat{\bar{x}}_{k+1}=\left(\mathcal{A}\left(\rho_{k}\right)-K\left(\rho_{k}\right) \mathcal{C}\right) \hat{\bar{x}}_{k}+\bar{B}\left(\rho_{k}\right) u_{k}+K^{A}\left(\rho_{k}\right) y_{k}+K\left(\rho_{k}\right) \Psi y_{k} \\
\hat{y}_{k}=\bar{C} \hat{\bar{x}}_{k}
\end{array}\right.
$$

with $\mathcal{A}\left(\rho_{k}\right)=\bar{A}\left(\rho_{k}\right)\left(I_{m}-\bar{F} \Pi \bar{C}\right)$ and $\mathcal{C}=\Psi \bar{C}$.

According to (7) and (15), the estimation error $e_{k}=\bar{x}_{k}-\hat{\bar{x}}_{k}$ and the residual $r_{k}$ are given by the following relations:

$$
\left\{\begin{array}{l}
e_{k+1}=\left(\mathcal{A}\left(\rho_{k}\right)-K\left(\rho_{k}\right) \mathcal{C}\right) e_{k}+\bar{F} \bar{f}_{k} \\
r_{k}=\bar{C} e_{k}
\end{array}\right.
$$

where $K\left(\rho_{k}\right)$ should be synthesized in order to guarantee the stability of the polytopic LPV filter and to achieve some error convergence performances. In fault-free case (i.e: $\bar{f}_{k}=0$ ), the previous relations are rewritten such that:

$$
\left\{\begin{array}{l}
\bar{e}_{k+1}=\left(\mathcal{A}\left(\rho_{k}\right)-K\left(\rho_{k}\right) \mathcal{C}\right) \bar{e}_{k} \\
\bar{r}_{k}=\bar{C} \bar{e}_{k}
\end{array}\right.
$$


where $\bar{e}_{k}$ and $\bar{r}_{k}$ denote respectively the estimation error and the residual in fault-free case.

Note that the residual $r_{k}$ in (16) is obtained by multiplying the estimation error $e_{k}$ by the matrix $\bar{C}$ and can be written as follows

$$
r_{k+1}=\bar{C} e_{k+1}=\bar{C}\left(\mathcal{A}\left(\rho_{k}\right)-K\left(\rho_{k}\right) \mathcal{C}\right) e_{k}+\bar{C} \bar{F} \bar{f}_{k}
$$

So, based on equation (17), it can be rewritten such as

$$
r_{k}=\bar{r}_{k}+\bar{C} \bar{F} \bar{f}_{k-1}
$$

Now, from equation (19), the polytopic LPV filter is able to give two different information by the use of a specific matrix $\left[\begin{array}{c}\Psi \\ \Pi\end{array}\right]$ such that a projected residual $p_{k}$ is defined as follows:

$$
p_{k}=\left[\begin{array}{c}
\Psi \\
\Pi
\end{array}\right] r_{k}=\left[\begin{array}{c}
\Psi \\
\Pi
\end{array}\right]\left(\bar{r}_{k}+\bar{C} \bar{F} \bar{f}_{k-1}\right)=\left[\begin{array}{c}
\Psi \bar{r}_{k}+\Psi \bar{C} \bar{F} \bar{f}_{k-1} \\
\Pi \bar{r}_{k}+\Pi \bar{C} \bar{F} \bar{f}_{k-1}
\end{array}\right]
$$

Then, by considering the following properties (which involve the gain decomposition (12)):

$$
\Pi \bar{C} \bar{F}=I \text { and } \Psi \bar{C} \bar{F}=0
$$

the generation of the projected residual $p_{k}$ from equation (20), can be defined as follows:

$$
p_{k}=\left[\begin{array}{c}
\Psi \\
\Pi
\end{array}\right] r_{k}=\left[\begin{array}{l}
\Psi \bar{r}_{k} \\
\Pi \bar{r}_{k}+\bar{f}_{k-1}
\end{array}\right]=\left[\begin{array}{l}
\gamma_{k} \\
\Omega_{k}
\end{array}\right]
$$

The residual $p_{k}$ is split into two sub-vectors: a vector insensitive to fault $\bar{f}_{k}$ noted $\gamma_{k}$ and a vector sensitive only to fault $\bar{f}_{k}$ noted $\Omega_{k}$. The vector 
insensitive to fault $\bar{f}_{k}\left(\gamma_{k} \in \mathbb{R}^{m-q}\right)$ is close to zero in fault-free case or in faulty case when the fault $\bar{f}_{k}$ occurs on the system. However, the vector $\gamma_{k}$ is different from zero meaning the presence of modeling errors or other sensor faults which are not considered in the synthesis of the filter. Such vector $\gamma_{k}$ is an indicator to qualify the efficiency of the polytopic LPV filter. If a fault $\bar{f}_{k}$ occurs on the system, a residual evaluation technique should be computed in order to indicate the accuracy of the projected residual vector $p_{k}$ and more precisely, the accuracy of the last component $\Omega_{k}=\Pi \bar{r}_{k}+\bar{f}_{k-1}$. With respect to the component $\Omega_{k} \in \mathbb{R}^{q}$ which represents the residual vector sensitive to fault, this one can be used to a fault estimation of $\bar{f}_{k}$. With only one sample for time delay and as mentioned in Proposition 1 in (Rodrigues et al., 2008), an estimation $\hat{f}_{k}$ of sensor fault $\bar{f}_{k}$ could be carried out through a Moore-Penrose matrix like:

$$
\hat{f}_{k}=(\bar{F})^{+} \bar{F} \Omega_{k}
$$

\subsection{Polytopic LPV filter stability}

The stability problem is reduced to find matrices gains $K_{j}, \forall j \in[1, \ldots, N]$ so that the gain interpolation $K\left(\rho_{k}\right)$ ensures the asymptotic convergence of the estimation error (17) in fault-free case to the origin. Another goal is to obtain less restrictive conditions of the synthesis than the commonly use of a single symmetric definite positive matrix. So, we will use the notion of poly-quadratic stability if there exists a parameter-dependent quadratic Lyapunov function as mentioned in (Anstett et al., 2009).

Proposition 1. The estimation error (17) is poly-quadratically stable if there 
exist symmetric positive definite matrices $P_{j}$, matrices $R_{j}$ and $G$ of appropriate dimensions $\forall j \in[1, \ldots, N]$ such that:

$$
\left(\begin{array}{ll}
-P_{j} & \mathcal{A}_{j}^{T} G-\mathcal{C}^{T} R_{j}^{T} \\
G \mathcal{A}_{j}-R_{j} \mathcal{C} & P_{i}-\left(G+G^{T}\right)
\end{array}\right)<0, \forall i, j \in[1, \ldots, N]
$$

Then, the gain $K_{j}$ is given by $K_{j}=G^{-1} R_{j}$.

Proof:

Let the Lyapunov function defined such as $V\left(\bar{e}_{k}, \rho_{k}\right)=\bar{e}_{k}^{T} P\left(\rho_{k}\right) \bar{e}_{k}>0$. To ensure stability, its difference $\mathcal{L}=V\left(\bar{e}_{k+1}, \rho_{k+1}\right)-V\left(\bar{e}_{k}, \rho_{k}\right)$ along the solution of equation (17) should be negative definite such that:

$$
\left(\mathcal{A}\left(\rho_{k}\right)-K\left(\rho_{k}\right) \mathcal{C}\right)^{T} P\left(\rho_{k+1}\right)\left(\mathcal{A}\left(\rho_{k}\right)-K\left(\rho_{k}\right) \mathcal{C}\right)-P\left(\rho_{k}\right)<0
$$

with $\mathcal{A}\left(\rho_{k}\right)=\sum_{j=1}^{N} \rho_{j}\left(\theta_{k}\right) \mathcal{A}_{j}$ and the Lyapunov matrix $P\left(\rho_{k}\right)=\sum_{j=1}^{N} \rho_{j}\left(\theta_{k}\right) P_{j}$ over convex set $\Lambda$.

Let's assume that (24) is true. Then, using notation $R_{j}=G K_{j}$, multiplying each inequality $(24)$ by $\rho_{j}\left(\theta_{k}\right)$ and adding all of them $\forall j \in[1, \ldots, N]$ with $\sum_{j=1}^{N} \rho_{j}\left(\theta_{k}\right)$, then $(24)$ becomes $\forall j \in[1, \ldots, N]$ :

$$
\left(\begin{array}{ll}
-\sum_{j=1}^{N} \rho_{j}\left(\theta_{k}\right) P_{j} & \sum_{j=1}^{N} \rho_{j}\left(\theta_{k}\right)\left(\mathcal{A}_{j}-K_{j} \mathcal{C}\right)^{T} G^{T} \\
G \sum_{j=1}^{N} \rho_{j}\left(\theta_{k}\right)\left(\mathcal{A}_{j}-K_{j} \mathcal{C}\right) & P_{i}-\left(G+G^{T}\right)
\end{array}\right)<0
$$

and by adding the inequalities (26) over $i,(26)$ becomes:

$$
\left(\begin{array}{ll}
-P\left(\rho_{k}\right) & \left(\mathcal{A}^{T}\left(\rho_{k}\right)-K\left(\rho_{k}\right) \mathcal{C}\right)^{T} G^{T} \\
G\left(\mathcal{A}\left(\rho_{k}\right)-K\left(\rho_{k}\right) \mathcal{C}\right) & P_{+}\left(\rho_{k}\right)-\left(G+G^{T}\right)
\end{array}\right)<0
$$


under the following notations:

$$
\begin{aligned}
P\left(\rho_{k}\right) & =\sum_{j=1}^{N} \rho_{j}\left(\theta_{k}\right) P_{j} \\
P_{+}\left(\rho_{k}\right) & =P\left(\rho_{k+1}\right)=\sum_{j=1}^{N} \rho_{j}\left(\theta_{k+1}\right) P_{j}=\sum_{i=1}^{N} \rho_{i}\left(\theta_{k}\right) P_{i}
\end{aligned}
$$

Multiplying (27) on the left by $\left[I\left(\mathcal{A}\left(\rho_{k}\right)-K\left(\rho_{k}\right) \mathcal{C}\right)^{T}\right]$ and on the right by $\left[I\left(\mathcal{A}\left(\rho_{k}\right)-K\left(\rho_{k}\right) \mathcal{C}\right)^{T}\right]^{T}$, the following inequality is obtained:

$$
\left(\mathcal{A}\left(\rho_{k}\right)-K\left(\rho_{k}\right) \mathcal{C}\right)^{T} P_{+}\left(\rho_{k}\right)\left(\mathcal{A}\left(\rho_{k}\right)-K\left(\rho_{k}\right) \mathcal{C}\right)-P\left(\rho_{k}\right)<0
$$

In other words, the inequality (29) is the same inequality as in (25) by recalling that $P_{+}\left(\rho_{k}\right)=P\left(\rho_{k+1}\right)$. Hence, the estimation error (17) satisfies the condition of poly-quadratic stability.

As explained in the previous paragraphs, the design of a stable single polytopic LPV filter has been provided to detect sensor faults. To achieve a fault diagnosis task, fault isolation, which consists in deciding which element(s) of the system has (have) indeed failed, a fault isolation procedure should be performed as defined in the next section.

\subsection{Fault isolation design}

As presented in Fig. (1), a polytopic LPV filter can detect, isolate and estimate sensor faults at each sample $k$ based on $\gamma_{k}$ and $\Omega_{k}$ vectors norm. However, the synthesis of a single filter to detect all sensor faults is not possible due to the rank condition like $\operatorname{rank}(\bar{C} \bar{F})=q<m$ which can not be completed. So, in this case, the method consists in designing a bank of $m$ polytopic LPV filters where each of them is synthesized to estimate faults. 
The idea presented here is based on the efficiency of the "quality factor" which is insensitive to specific faults vector but sensitive to others.

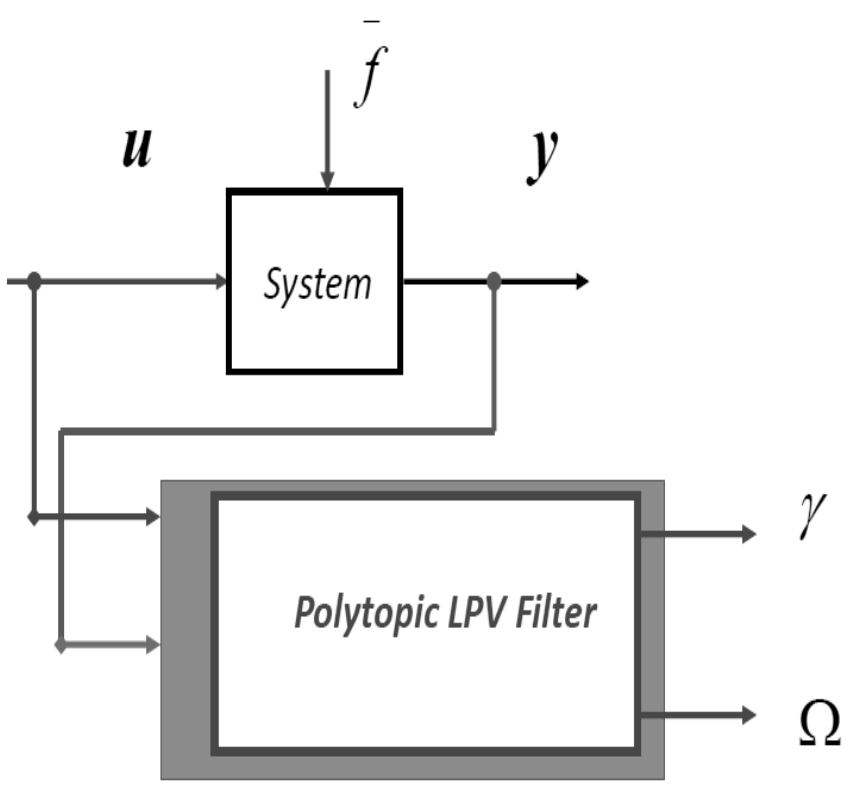

Figure 1: Polytopic filter scheme

This fault diagnosis scheme is similar to the well-known generalized residual structure approach. According to the developed filter, it was possible to establish various bank of filters such as Generalized Observer Scheme (GOS) only able to isolate one fault or Dedicated Observer Scheme (DOS) able to isolate simultaneous faults as proposed by (Frank and Ding, 1997). For the well-known GOS, each residual $\gamma^{i}, i \in[1, \ldots, m]$ of the $i_{t h}$ polytopic filter is insensitive to the $i_{t h}$ sensor fault but sensitive to all other faults i.e $\gamma_{k} \in \mathbb{R}^{m-1}$ and $\Omega_{k} \in \mathbb{R}^{1}$. However, for DOS, each residual $\gamma^{i}, i \in[1, \ldots, m]$ of the $i_{t h}$ polytopic filter is insensitive to all sensor faults except the $i_{t h}$ sensor. i.e $\gamma_{k} \in \mathbb{R}^{1}$ and $\Omega_{k} \in \mathbb{R}^{m-1}$. Fig. (2) summarizes the different levels of the FDI 
scheme.

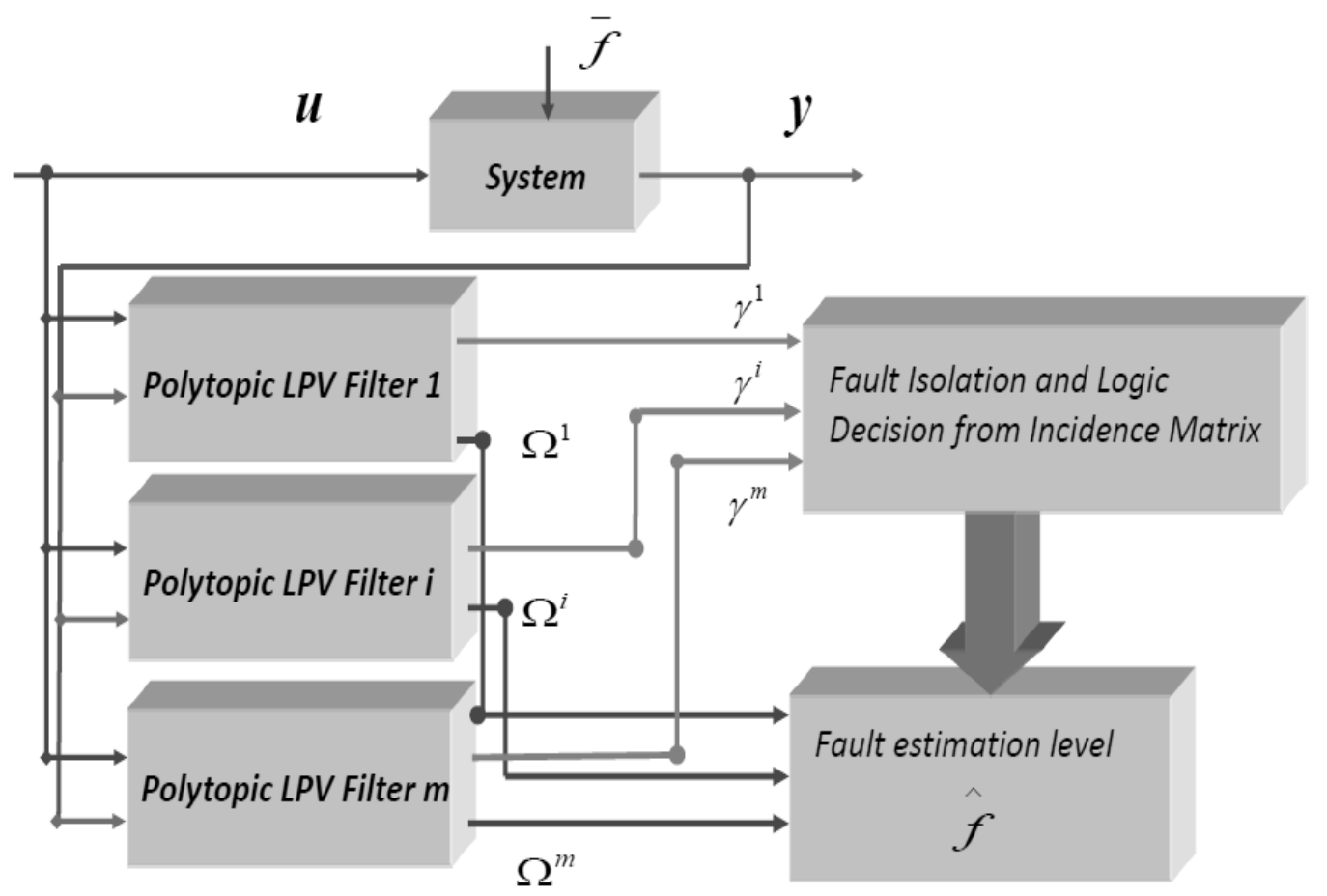

Figure 2: FDI scheme for Isolation purpose

An incidence matrix (Tables 1 or 2 ) can be established where $\left\|\gamma_{k}\right\|$ is considered equal to ' 0 ' when the residual is close to zero in some sense and equal to ' 1 ' otherwise. Each column of the incidence matrix is called the coherence vector associated with each fault signature. From Tables 1 or 2 , a decision making selects which ones of the sensor is 'faulty'. A column indicates that one signal is equal to zero whereas the others are equal to one, i.e the ' 0 ' indicates the localization of the sensor fault with the dedicated polytopic LPV filter.

Decision making is then used according to elementary logic (Leonhardt 
and Ayoubi, 1997), which can be described as follows: a 'fault indicator' is equal to one if the residual vector $\Upsilon=\left[\begin{array}{c}\left\|\gamma_{k}\right\|^{1} \\ \cdots \\ \left\|\gamma_{k}\right\|^{i} \\ \cdots \\ \left\|\gamma_{k}\right\|^{m}\end{array}\right]$ generated by the bank, is equal to a column of the incidence matrix, and to zero otherwise. The element associated with the indicator being equal to one is then declared to be 'faulty'.

Table 1: Incidence matrix - GOS $(*)$ Other fault or minimum two sensors are faulty.

\begin{tabular}{|c|c|c|c|c|c|c|c|}
\hline Residual & No Fault & $\bar{f}^{1}$ & $\ldots$ & $\bar{f}^{i}$ & $\ldots$ & $\bar{f}^{m}$ & $*$ \\
\hline$\left\|\gamma_{k}\right\|^{1}$ & 0 & $\mathbf{0}$ & 1 & 1 & 1 & 1 & 1 \\
$\vdots$ & $\vdots$ & 1 & $\ddots$ & $\vdots$ & $\vdots$ & $\vdots$ & 1 \\
$\left\|\gamma_{k}\right\|^{i}$ & 0 & 1 & $\vdots$ & $\mathbf{0}$ & $\vdots$ & 1 & 1 \\
$\vdots$ & $\vdots$ & $\vdots$ & $\vdots$ & $\vdots$ & $\ddots$ & $\vdots$ & 1 \\
$\left\|\gamma_{k}\right\|^{m}$ & 0 & 1 & $\ldots$ & 1 & $\ldots$ & $\mathbf{0}$ & 1 \\
\hline
\end{tabular}

Table 2: Incidence matrix - DOS $\left(^{*}\right)$ Other fault or all sensors are faulty.

\begin{tabular}{|c|c|c|c|c|c|c|c|}
\hline Residual & No Fault & $\bar{f}^{1}$ & $\ldots$ & $\bar{f}^{i}$ & $\ldots$ & $\bar{f}^{m}$ & $*$ \\
\hline$\|\gamma\|^{1}$ & 0 & $\mathbf{1}$ & 0 & 0 & 0 & 0 & 1 \\
$\vdots$ & $\vdots$ & 0 & $\ddots$ & $\vdots$ & $\vdots$ & $\vdots$ & 1 \\
$\|\gamma\|^{i}$ & 0 & 0 & $\vdots$ & $\mathbf{1}$ & $\vdots$ & 0 & 1 \\
$\vdots$ & $\vdots$ & $\vdots$ & $\vdots$ & $\vdots$ & $\ddots$ & $\vdots$ & 1 \\
$\|\gamma\|^{m}$ & 0 & 0 & $\ldots$ & 0 & $\ldots$ & $\mathbf{1}$ & 1 \\
\hline
\end{tabular}

Note: The Fault Isolation method is already based on the fact that only 
one fault can be detected at exactly the same time, for recovering fault signature from the Tables 1 or 2 . It should be noticed that the probability that two or more faults appearing at exactly the same time, so simultaneously, is close to zero. Multiple faults can appear during an experiment, but not exactly at the same time. If multiple faults appear at exactly the same time (very small probability), then the fault isolation is not guaranteed but the fault detection is still working.

\section{The winding machine}

\subsection{System description}

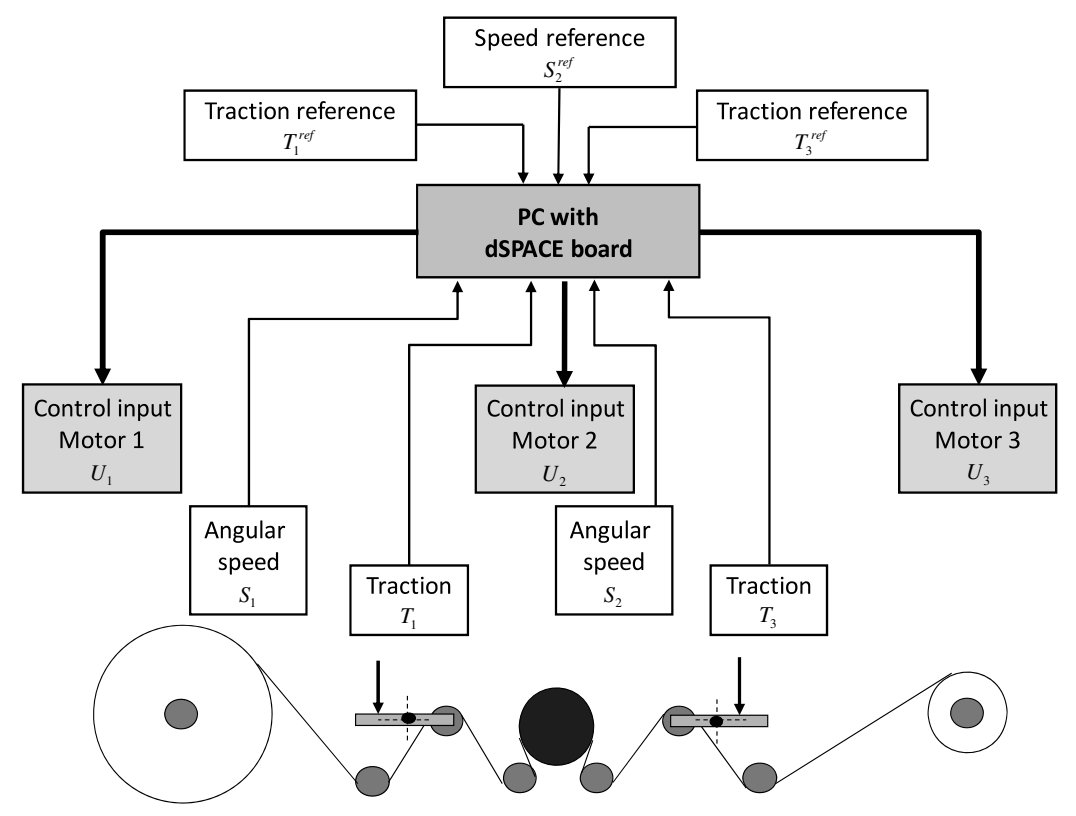

Figure 3: Winding process control architecture 
The winding process, (Noura et al., 2009) in chapter 3, is composed of a plastic web and three reels, respectively, called the unwinding, pacer and rewinding reels but the radius is unmeasurable directly. Each reel is coupled with a DC-motor via gear reduction. The angular speed of the first two reels $\left(S_{1}, S_{2}\right)$ and both the tensions between the reels $\left(T_{1}, T_{3}\right)$ are measured by tachometers and tension meters. The three control inputs $U_{1}, U_{2}$ and $U_{3}$ are the input voltage of the three motors as illustrated in Fig.3. Each motor is driven by a local controller composed of one or two PI controllers (Ponsart et al., 2010). The first control loop adjusts the motor current $\left(I_{1}, I_{2}, I_{3}\right)$, and its constant of time integration is about $40 \mathrm{~ms}$, while the second loop controls the angular speed with an integration time constant equal to $200 \mathrm{~ms}$. The set-points of those controllers are computed by a Programmable Logic Controller (PLC) in order to control both tensions and the linear velocity of the strip (300 $\mathrm{m}$ lentgh, $5 \mathrm{~cm}$ broad and $0.2 \mathrm{~mm}$ thickness). Under specific experimental investigation which lasts $40 \mathrm{~min}$, the radius of the unwinding reel varies from 210 to $70 \mathrm{~mm}$. A real-time development environment (Simulink Real-Time Workshop+dSPACE) based on a PC computer is used instead of the PLC to improve control for instance. System inputs and outputs are given in the interval $[0100 \%]$ corresponding to $[-10 \mathrm{~V} ; 10 \mathrm{~V}]$ equal to $[-1 ;+1]$ with Matlab variables.

\subsection{The winding machine modelization with sensor faults}

Based on (Ponsart et al., 2010), where identification and validation steps are developed and presented in details, the winding machine can be repre- 
sented in the following state space representation:

$$
\left\{\begin{array}{l}
x_{k+1}=A\left(R_{k}\right) x_{k}+B\left(R_{k}\right) u_{k} \\
y_{k}=C x_{k}+F f_{k}
\end{array}\right.
$$

where $x_{k}=x\left(k T_{e}\right)$, with the sampling period $T_{e}=0.1 s, y_{k}=x_{k}=\left[T_{1} S_{2} T_{3}\right]^{T}$ and $u_{k}=\left[\begin{array}{lll}U_{1} & U_{2} & U_{3}\end{array}\right]^{T}$. The system matrix $A$ and the control matrix $B$ are linear polynomial matrices depending on a bounded positive time varying parameter noted $R_{k}$ where $0<R_{\min }<R_{k}<R_{\max }$ and verifying:

$$
G(R)=G_{0} R^{0}+G_{1} R^{1}+G_{2} R^{2}+\ldots+G_{\alpha} R^{\alpha}
$$

where $G$ stands for $A$ or $B, \alpha$ defines the polynomial degree equal to 6 as proof in (Theilliol et al., 2008) . Here, the method developed by (Hetel et al., 2007) is considered. This method underlines the exact equivalence between polynomial model and convex polytopic representation. Thus each polynomial matrix is defined by a convex polytope with $(\alpha+1)$ vertices $\Delta_{j}^{G}$ calculated as follows:

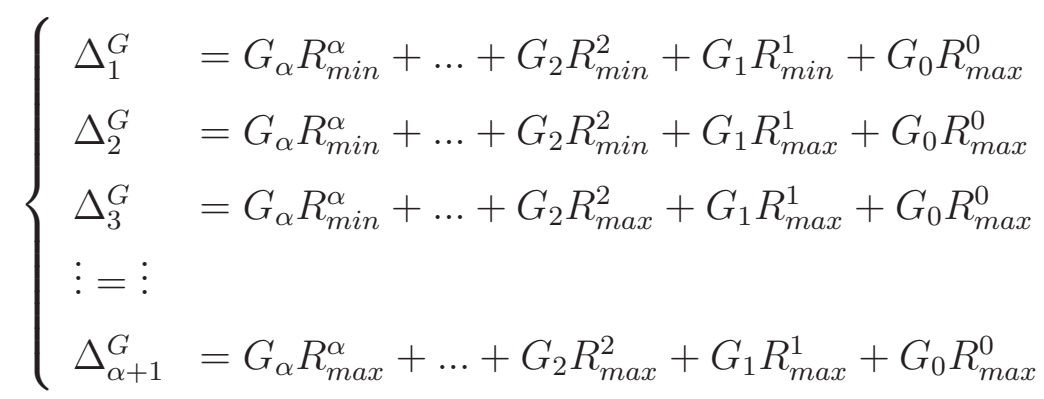

The convex polytope formulation is achieved by the computation of the parameter $\rho_{j}(R)(\forall j \in[0, \ldots, \alpha])$ by the following recursive algorithm with 
$\tau=2, \ldots, \alpha:$

$$
\left\{\begin{array}{l}
\rho_{1}(R)=1-\frac{R-R_{\min }}{R_{\max }-R_{\min }} \\
\rho_{\alpha+1}(R)=\frac{R^{\alpha}-R_{\min }^{\alpha}}{R_{\max }^{\alpha}-R_{\min }^{\alpha}} \\
\rho_{\tau}(R)=\frac{R^{\tau-1}-R_{\min }^{\tau-1}}{R_{\max }^{\tau-1}-R_{\min }^{\tau-1}}-\sum_{j=\tau+1}^{\alpha+1} \rho_{j}(R)
\end{array}\right.
$$

Therefore, the parameter $\rho_{j}(R)$ lies in a specific convex set $\Lambda$ :

$$
\Lambda=\left\{\rho(R) \in \mathbb{R}^{\alpha+1}, \rho=\left[\rho_{1} \ldots \rho_{\alpha+1}\right]^{T} \text { and } \sum_{j=1}^{\alpha+1} \rho_{j}(R)=1 \rho_{j} \geq 0\right\}
$$

Whereupon, $\forall j \in[1, \ldots, \alpha+1], \Delta_{j}^{G}$ defines the vertices of a convex polytope such as:

$$
G(R)=\sum_{j=1}^{\alpha+1} \rho_{j}(R) \Delta_{j}^{G}
$$

Based on these previous equations (6) and (34), the faulty discrete state space representation (30) can be expressed as an augmented polytopic LPV system:

$$
\left\{\begin{aligned}
\bar{x}_{k+1} & =\sum_{j=1}^{\alpha+1} \rho_{j}\left(R_{k}\right)\left(\bar{A}_{j} \bar{x}_{k}+\bar{B}_{j} u_{k}+\bar{F} \bar{f}_{k}\right) \\
y_{k} & =\bar{C} \bar{x}_{k}
\end{aligned}\right.
$$

where $\bar{A}_{j} \in \mathbb{R}^{n \times n}$ and $\bar{B}_{j} \in \mathbb{R}^{n \times p}$ are constant matrices and $\forall j \in[1, \ldots, \alpha+$ 1] : $\rho_{j} \geq 0, \sum_{j=1}^{\alpha+1} \rho_{j}\left(R_{k}\right)=1$ and $0<R_{\text {min }}<R_{k}<R_{\text {max }}$.

Here, the winding machine can be represented with $\alpha=6$ with a dependance on the unwinding real radius $R_{k}$ as in equation (35) with $0<R_{\min }<$ $R_{k}<R_{\max }$ with $R_{\min }=70 \mathrm{~mm}$ and $R_{\max }=210 \mathrm{~mm}$. For technical reasons, 
the radius is estimated via the following equation:

$$
R_{k}=R_{k-1}+\frac{h}{2 \pi} S_{1, k}
$$

where $h$ is the strip thickness.

The measured outputs are $y_{k}=x_{k}=\left[\begin{array}{lll}T_{1} & S_{2} & T_{3}\end{array}\right]^{T} . \quad S_{1, k}$ is the time dependance of the angular speed of reel $S_{1}$ and is assumed to be fault-free.

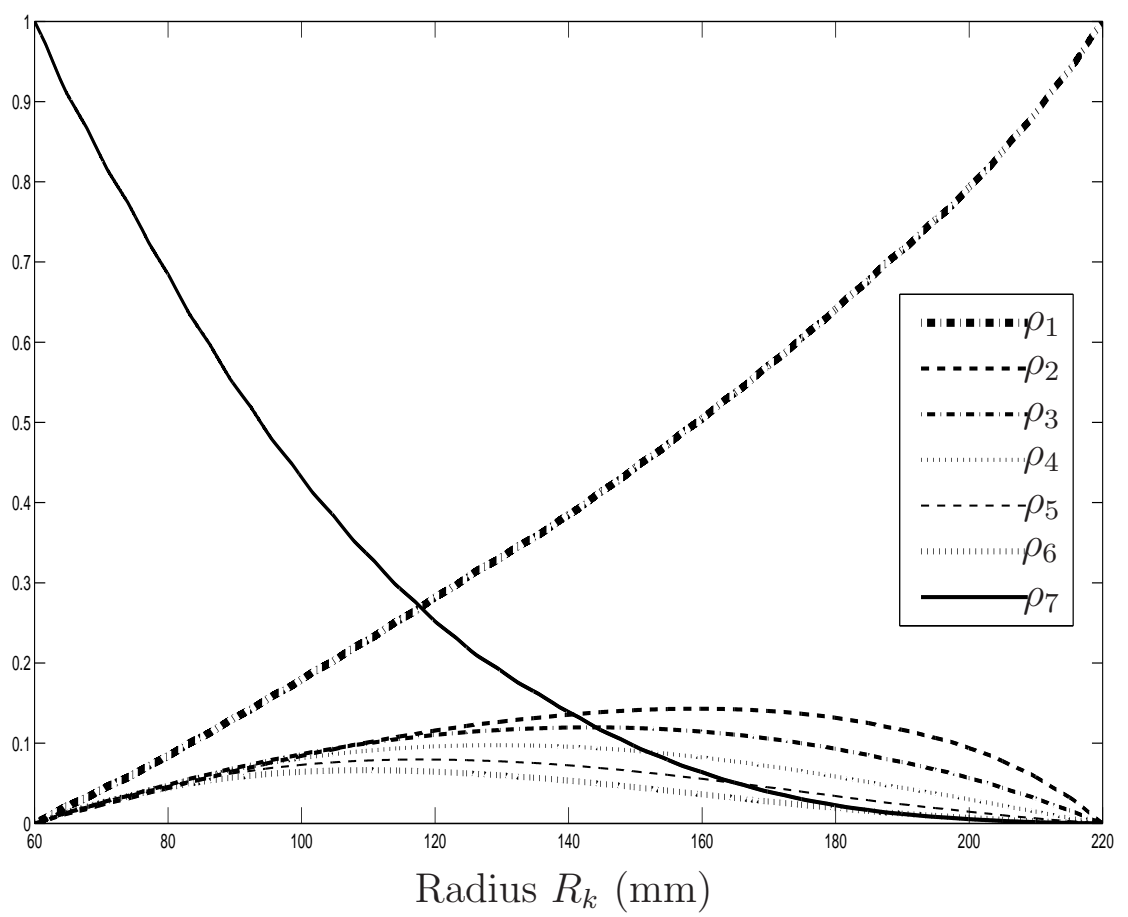

Figure 4: Parameters evolution $\rho_{j}$

Fig. (4) represents the $6+1$ parameters evolution $\rho_{j}$ which have been obtained for this example. Their evolutions justify the use of a LPV model where parameters evolve in a nonlinear way. The different matrices $A_{j}$ and $B_{j}$ are presented in Appendix. 
Note that an input-output linearizing control law, based on the polynomial model, has been implemented (as in (Noura et al., 2009)) using nonlinear theoretical development from (Fossard and Normand-Cyrot, 1995) and (Isidori, 1995): it is essential since this system is unstable in open loop. It can be verified through the computation of eigenvalues of various matrices $A_{j}$ presented in Appendix. Without any restrictions on the developed method, we consider in the following of the paper that only one fault can occur on the system. In order to evaluate the method, a bank of polytopic LPV filters have been developed in order to detect, isolate and estimate an accurate state of the system in presence of faults. Each one of these filters is dedicated for only one specific sensor fault following the GOS principle.

Remark 1. If two faults are considered to occur simultaneously, the decoupling condition is verified $(\operatorname{rank}(\bar{C} \bar{F})=q=2<3)$. A bank of 3 filters is established in order to provide a DOS structure $\left(\gamma_{k} \in \mathbb{R}^{2}\right.$ and $\left.\Omega_{k} \in \mathbb{R}^{1}\right)$. With such scheme, it is possible to detect, isolate and estimate two simultaneous faults under the assumption that no other faults occur or modeling errors. However if three faults occur, the proposed scheme is able to detect faults only. It can be noted that a single filter for all sensor faults with $F=I_{3}$ can not be synthesized as the decoupling condition $\operatorname{rank}(\bar{C} \bar{F})=q=3<m$ would not be fulfilled.

\subsection{Experimental results}

The effectiveness and performances of the developed FDI method is applied and a generalized polytopic LPV scheme is tested for the real process. The synthesis of LPV filter gains $K_{j}$, presented in Appendix, has been com- 
puted with the LMI Toolbox of the Robust Control Toolbox which is a part of the Matlab software environment.

In fault-free case: Fig.5 shows the evolution of the outputs driven in closed loop by the inputs in the fault-free case. This experiment was carried out for step changes with $20 \%$ of their corresponding operating values.

a)

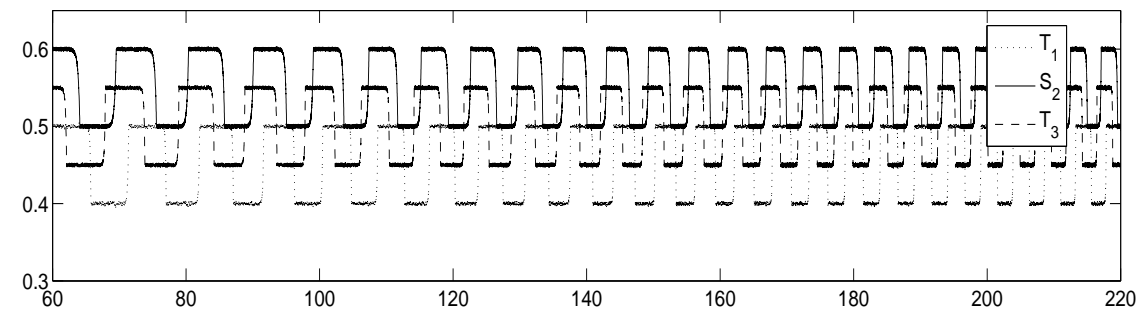

b)

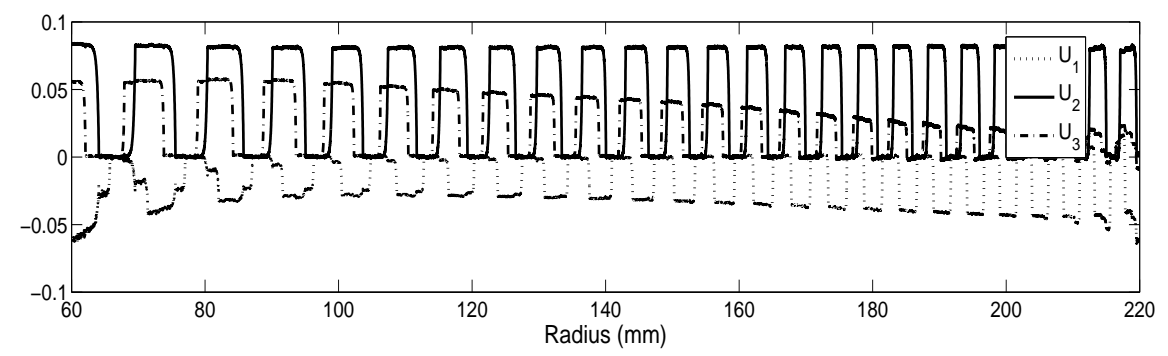

Figure 5: Fault-free case: a) System outputs b) System inputs

It should be noticed that the residuals generated by the polytopic filter depicted on Fig.6 are zero-mean: that means there are no fault and no modelings errors exist.

In the faulty case:

* First experimentation: A fault on the tension $T_{1}$ is considered to occur and to disappear at different times. According to (30), $F$ is equal to $\left[\begin{array}{lll}1 & 0 & 0\end{array}\right]^{T}$. As presented in Fig.7, the output $T_{1}$ is different from the nominal regime 

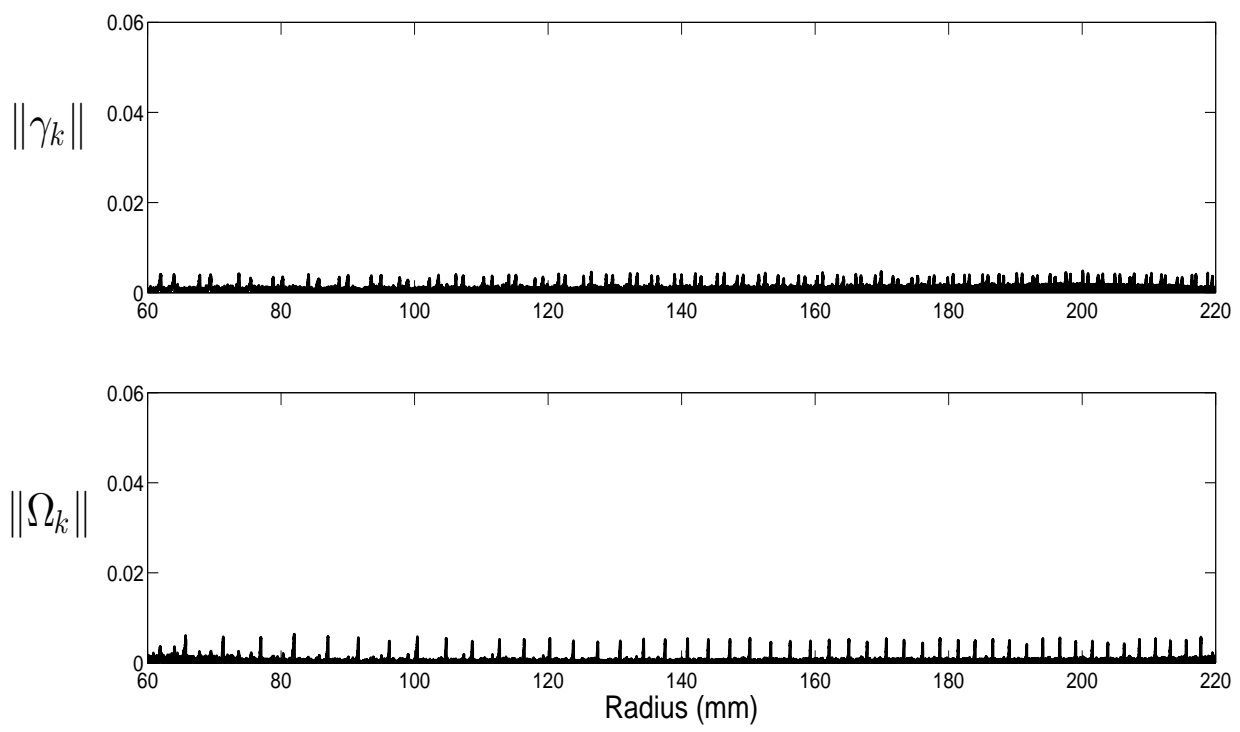

Figure 6: Residual vector norms in fault-free case

due to the presence of faults. The real output is different from the reference input for radius values around: 100, 170 and $215 \mathrm{~mm}$.

The results of the decoupling filter are depicted on Fig.8. As shown in Fig. 8 the residual $\left\|\Omega_{k}\right\|$ is affected by abrupt changes which correspond to the sensor fault of $T_{1}$. Such residual has been synthesized in order to be only sensitive to fault on the output $T_{1}$. Fig.8, it clearly appears that the quality factor $\left\|\gamma_{k}\right\|$ is equal to zero then the fault estimation derived from the sensitive residual vector can be used to estimate the real fault. The accurate fault magnitude estimation illustrates the performance and the effectiveness of the decoupling filter as it can be observed in Fig.9.

Remark 2. According to the developed MIMO control law, the output measurements are affected by step variation on the other ones. Consequently, 

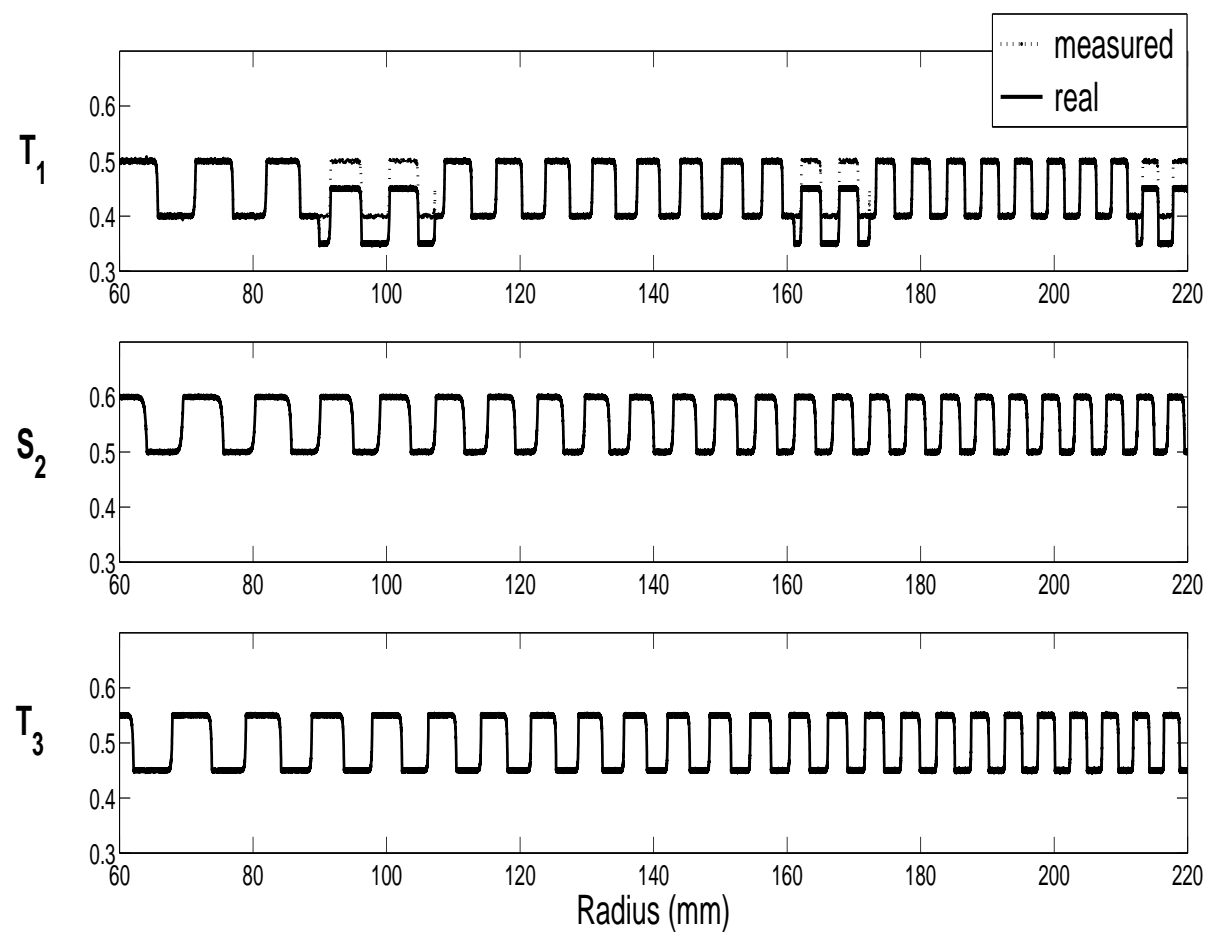

Figure 7: Measured and real system outputs in faulty case

some peaks can be observered on Fig.9 and other figures.

* Second experimentation: A sensor fault on the reel $S_{2}$ is considered. In order to underline the role of quality factor, the same filter, synthesized for the sensor fault occurrence on $T_{1}$, is used. So, it is not the adapted filter for sensor fault on $S_{2}$ and of course, the sensor fault estimation should be corrupted.

Fig.10 presents two residual norms generated by the considered polytopic LPV filter. As expected and illustrated in Fig.10, the residual $\left\|\gamma_{k}\right\|$ is not equal to zero which means that the residual $\left\|\Omega_{k}\right\|$ can not be considered to estimate the fault magnitude. 

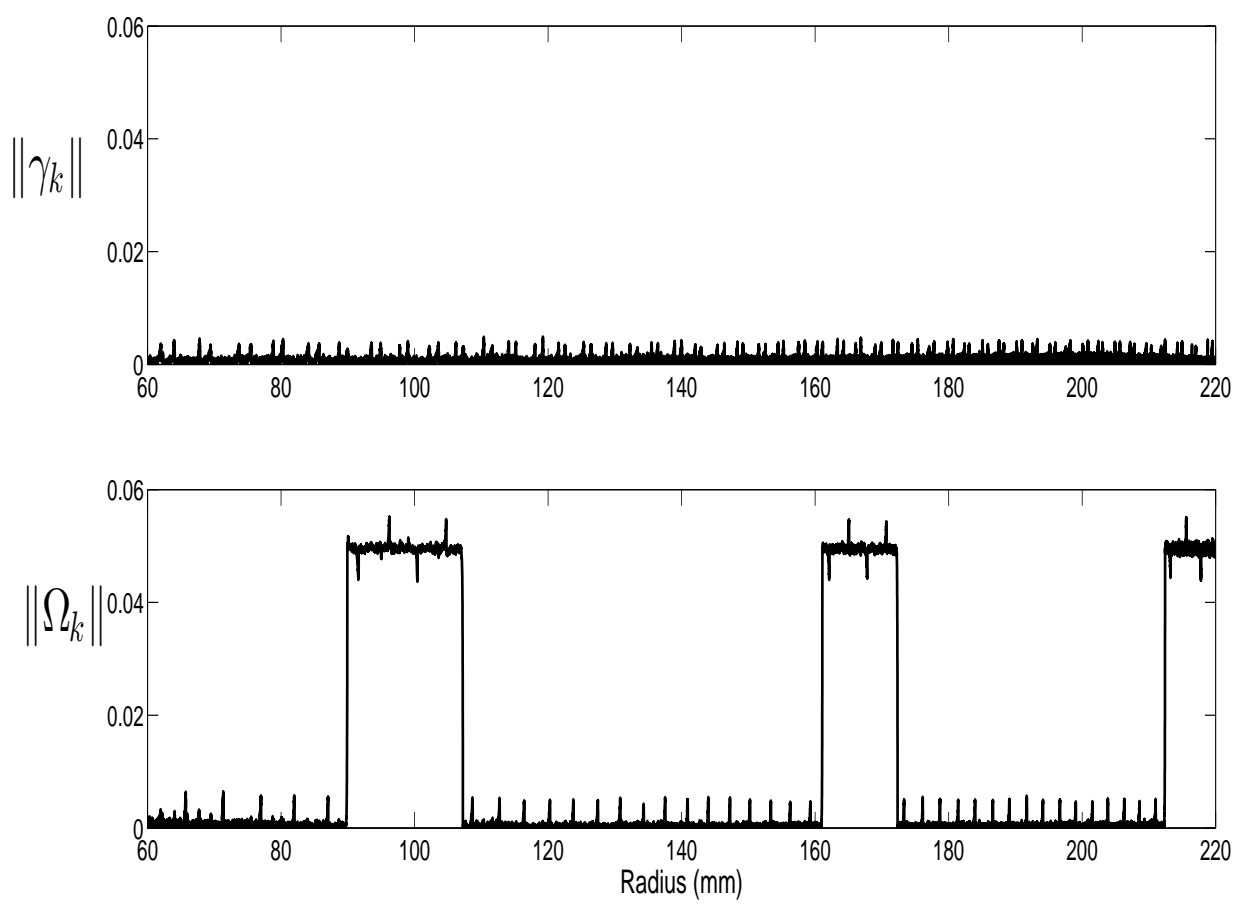

Figure 8: Bias on sensor $T_{1}$ - Norm of residuals filter designed for a fault on sensor $T_{1}$.

Now, consider the filter dedicated to the sensor fault on the reel $S_{2}$, i.e. synthesized with $F=\left[\begin{array}{lll}0 & 1 & 0\end{array}\right]^{T}$. As illustrated in Fig.11, such filter provides an appropriate sensor fault estimator. The quality factor equal to zero, indicates that the sensitive residual can be used to estimate this sensor fault (as in Table 1). The sensor fault estimation is close to the real one as shown in Fig.12.

The main advantage of this polytopic LPV filter is the performance of fault magnitude estimation but also the ability to generate an additional qualitative information from the insensitive residual: if this quality factor $\left\|\gamma_{k}\right\|$ is not equal to zero it means that it is corrupted by another fault or by 

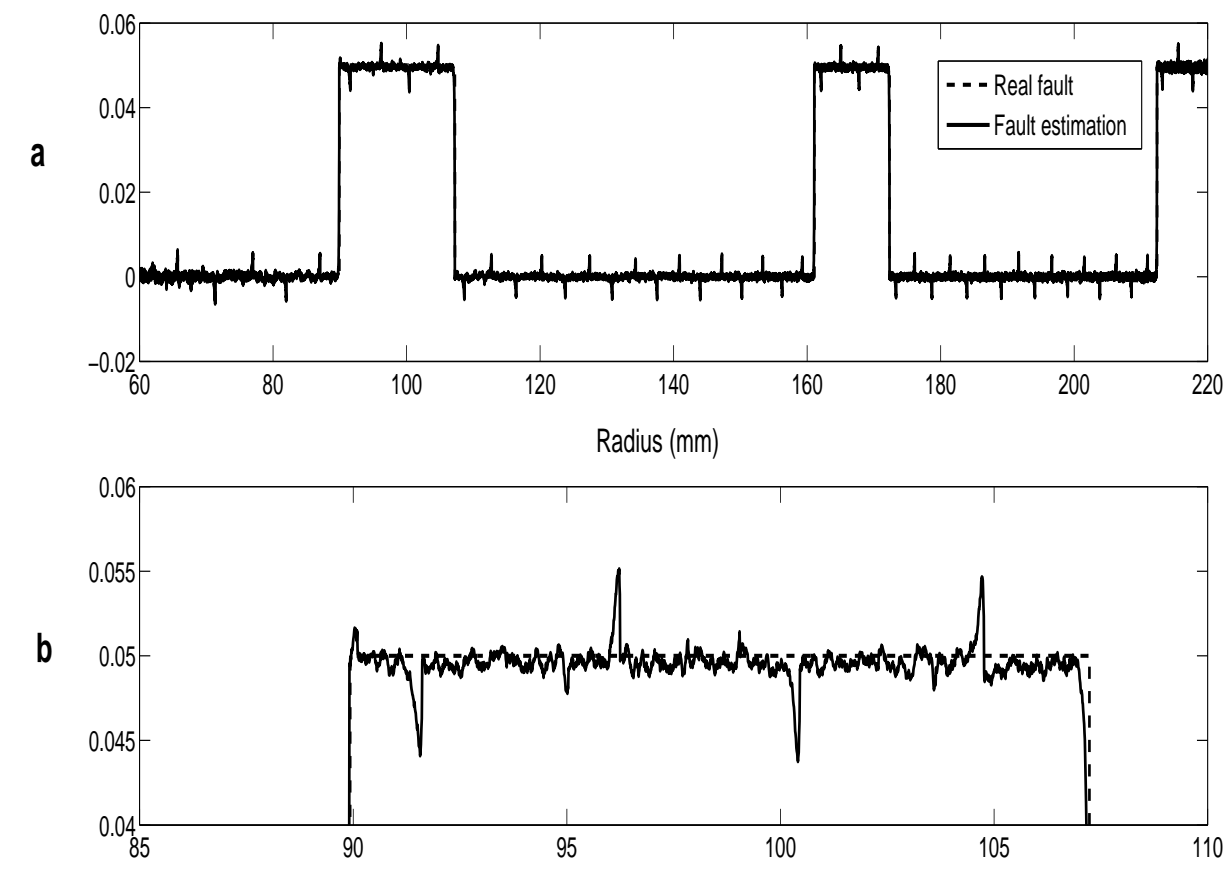

Figure 9: a) Sensor fault estimation $T_{1}$ b) Zoom around $R \approx 100 \mathrm{~mm}$ - Bias on sensor $T_{1}$.

modeling errors and could not be used to estimate a sensor fault magnitude.

\section{Conclusion}

In this paper, a general approach for sensor fault diagnosis designed for polytopic LPV systems has been proposed. This global method was developed using a polytopic LPV filter which is designed to detect, isolate and estimate sensor faults through a polytopic LPV representation. Even if a single filter synthesis is not always possible, a bank of dedicated polytopic LPV filters has been introduced to allow a detection, isolation and sensor fault 

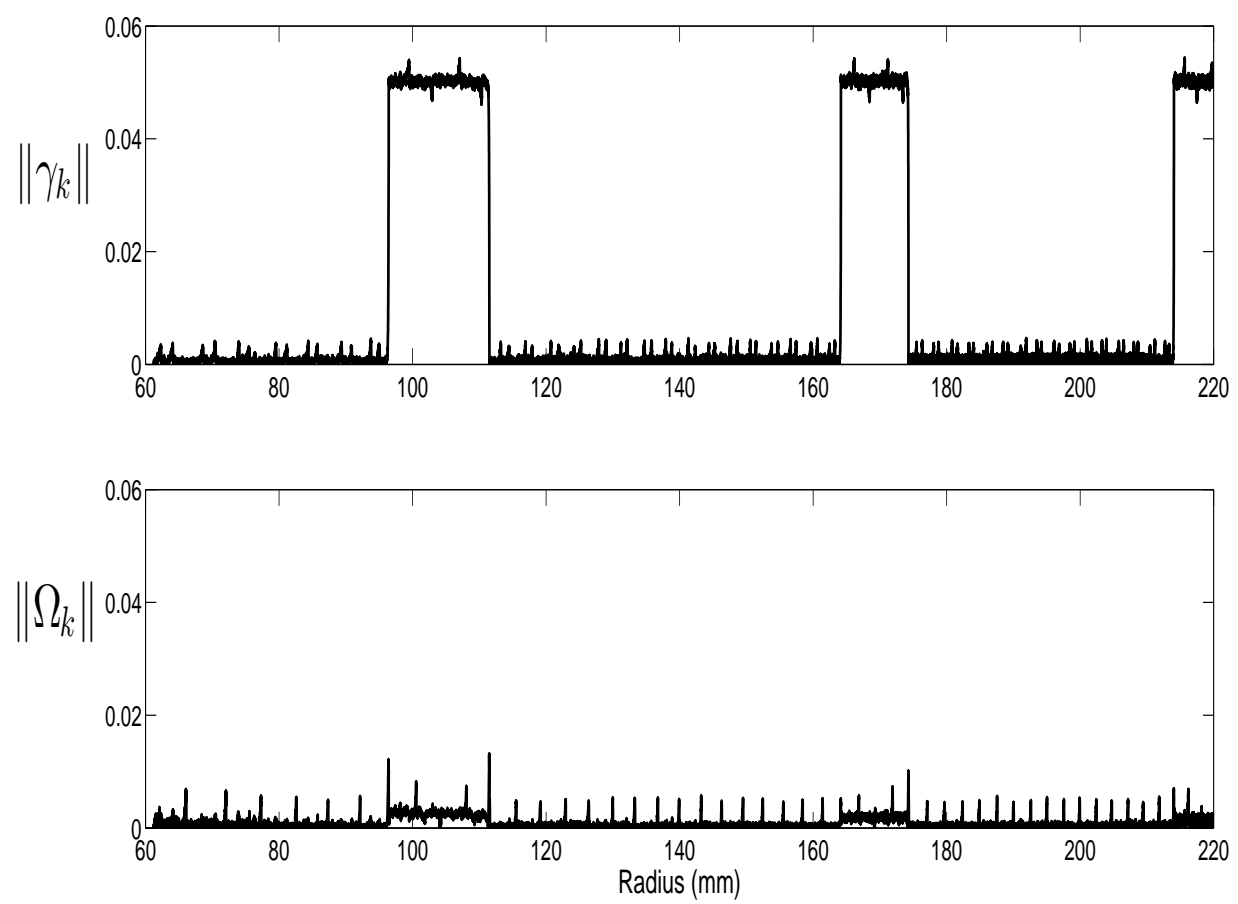

Figure 10: Bias on sensor $S_{2}$ - Norm of residuals filter designed for a fault on the output $T_{1}$.

estimation under a quality factor. The stability of this filter has been performed using LMI into a convex set. The effectiveness and performances of the polytopic LPV filter have been demonstrated in a web transport process: a winding machine. 

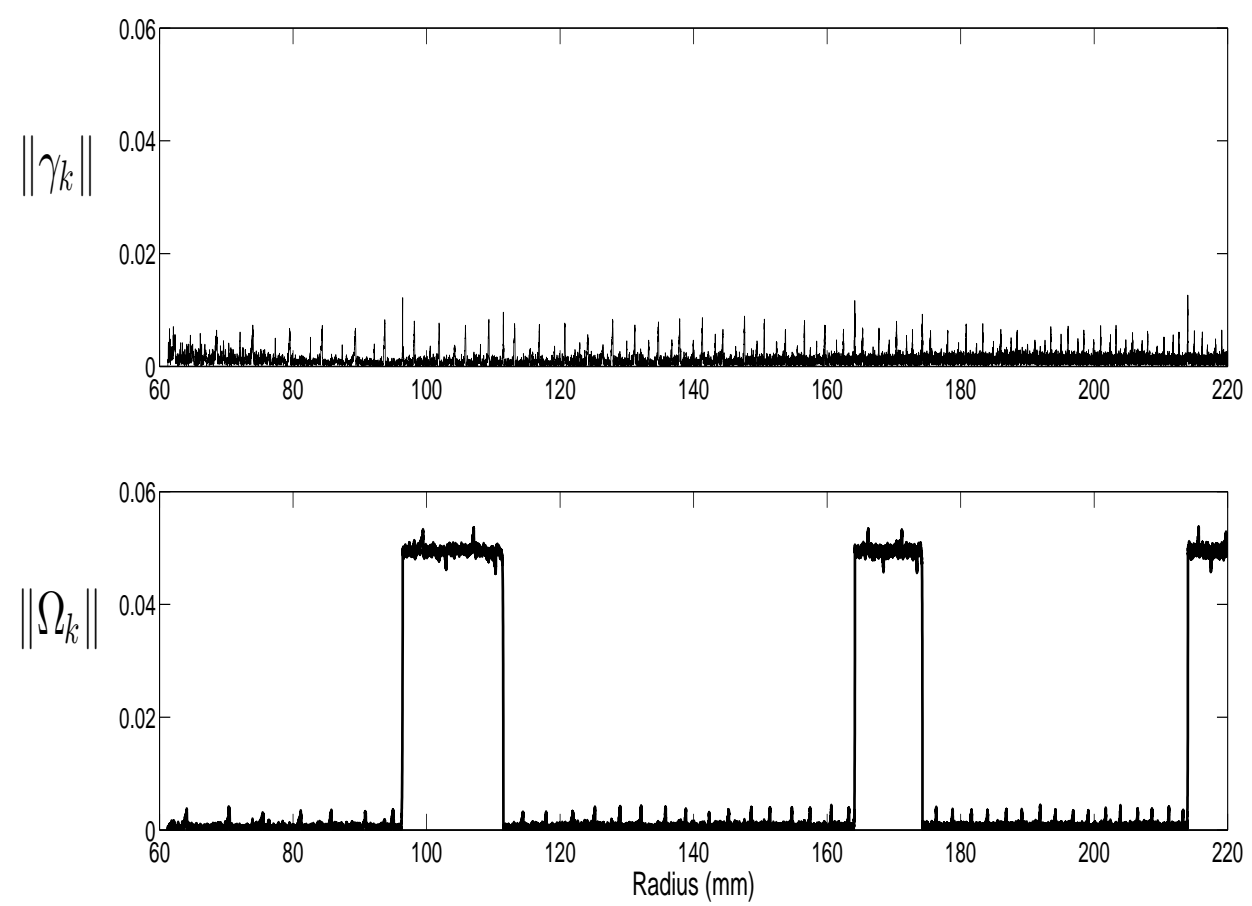

Figure 11: Bias on sensor $S_{2}$ - Norm of residuals filter designed for a fault on the output $S_{2}$.

\section{Appendix}

Matrices $A_{j}$ :

$$
\begin{aligned}
& A_{1}=\left(\begin{array}{ccc}
0.7175 & -0.8396 & -0.2270 \\
0.0122 & 0.5370 & -0.0650 \\
-0.0074 & 0.1675 & 0.1563
\end{array}\right) \\
& A_{2}=\left(\begin{array}{ccc}
29.7488 & -88.2373 & -29.5144 \\
-0.1112 & 6.9024 & -2.0532 \\
15.9178 & 126.3875 & -106.7138
\end{array}\right)
\end{aligned}
$$



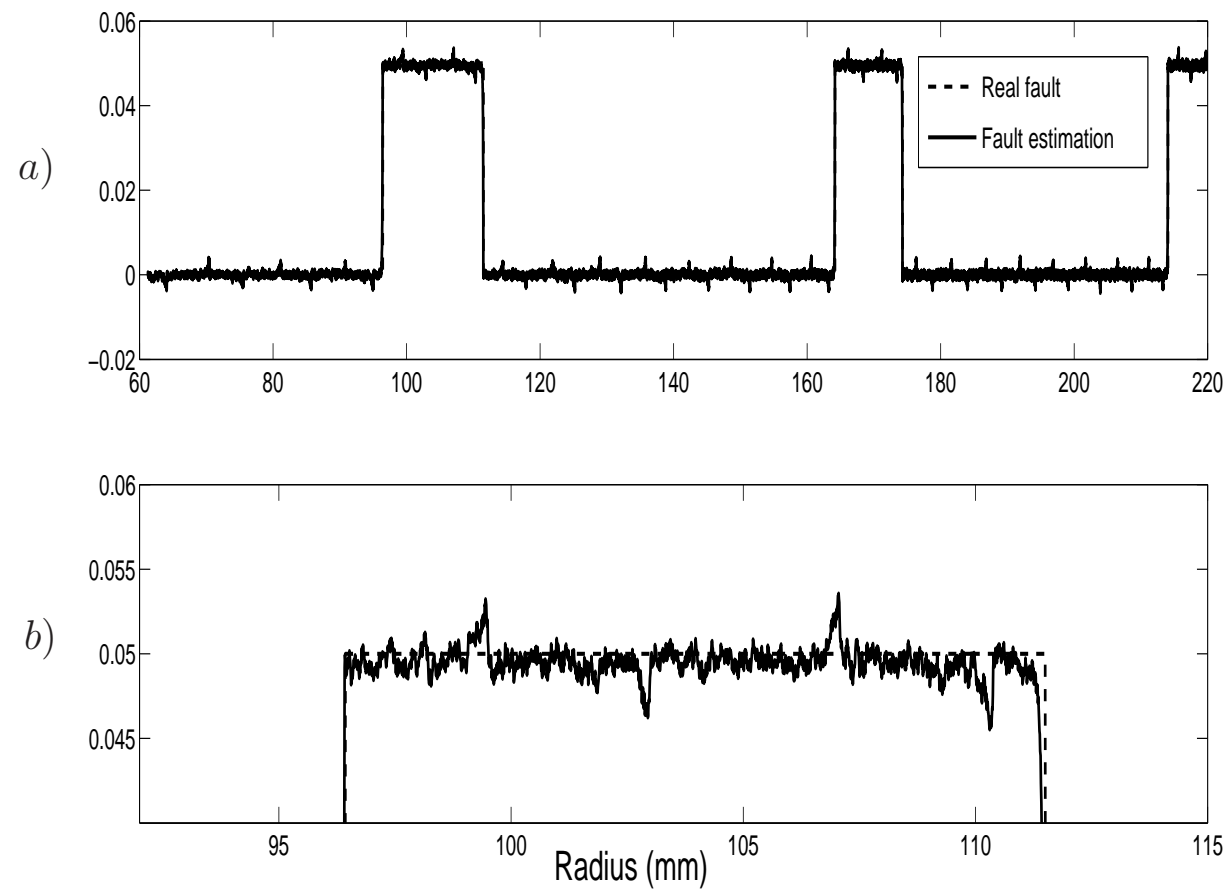

Figure 12: Bias on sensor $S_{2}$ - a) Sensor fault estimation $S_{2}$ b) Zoom around $R \approx 100 \mathrm{~mm}$.

$$
\begin{aligned}
& A_{3}=\left(\begin{array}{ccc}
-120.5489 & 485.6371 & 164.0216 \\
-0.6368 & -32.7339 & 8.5105 \\
-85.5748 & -665.5708 & 544.8643
\end{array}\right) \\
& A_{4}=\left(\begin{array}{ccc}
0.1947 & -1.0040 & -0.3470 \\
0.0037 & 0.0681 & -0.0156 \\
0.1841 & 1.3995 & -1.1048
\end{array}\right) \times 10^{3} \\
& A_{5}=\left(\begin{array}{lll}
-0.1444 & 0.9396 & 0.3338 \\
-0.0057 & -0.0625 & 0.0138 \\
-0.1848 & -1.3627 & 1.0353
\end{array}\right) \times 10^{3}
\end{aligned}
$$




$$
\begin{aligned}
& A_{6}=\left(\begin{array}{ccc}
43.2658 & -335.2822 & -121.1661 \\
3.1253 & 22.9693 & -4.9550 \\
72.3623 & 511.4675 & -369.7696
\end{array}\right) \\
& A_{7}=\left(\begin{array}{ccc}
0.4412 & -0.0026 & -0.0254 \\
0.0354 & 0.5204 & -0.0087 \\
-0.0471 & 0.3558 & 0.5210
\end{array}\right) \\
& \text { Matrices } B_{j} \text { : } \\
& B_{1}=\left(\begin{array}{ccc}
-1.1003 & 0.7929 & 0.3777 \\
0.0617 & 0.5755 & 0.1169 \\
-0.0470 & -0.2363 & 1.5299
\end{array}\right) \\
& B_{2}=\left(\begin{array}{ccc}
44.0344 & 122.2789 & -14.1984 \\
7.0243 & -11.0918 & 14.4880 \\
50.8487 & -128.2938 & 320.7654
\end{array}\right) \\
& B_{3}=\left(\begin{array}{ccc}
-0.1944 & -0.6379 & 0.0433 \\
-0.0328 & 0.0600 & -0.0715 \\
-0.2587 & 0.6670 & -1.6587
\end{array}\right) \times 10^{3} \\
& B_{4}=\left(\begin{array}{ccc}
0.2430 & 1.2760 & -0.0462 \\
0.0644 & -0.1180 & 0.1445 \\
0.5287 & -1.3791 & 3.4280
\end{array}\right) \times 10^{3} \\
& B_{5}=\left(\begin{array}{ccc}
-0.0885 & -1.1601 & 0.0115 \\
-0.0592 & 0.1092 & -0.1358 \\
-0.5074 & 1.3148 & -3.2489
\end{array}\right) \times 10^{3} \\
& B_{6}=\left(\begin{array}{ccc}
-0.0128 & 0.4023 & 0.0033 \\
0.0215 & -0.0374 & 0.0496 \\
0.1914 & -0.4814 & 1.1763
\end{array}\right) \times 10^{3}
\end{aligned}
$$




$$
B_{7}=\left(\begin{array}{ccc}
-1.1713 & 0.0450 & 0.2243 \\
0.0799 & 0.5809 & 0.0942 \\
-0.2549 & -0.5926 & 3.0868
\end{array}\right)
$$

Matrices $K_{j}$ for a fault vector equal to $F=\left[\begin{array}{lll}1 & 0 & 0\end{array}\right]^{T}$ :

$$
\begin{aligned}
& K_{1}=\left(\begin{array}{ccc}
0.6663 & 0 & -0.3041 \\
0.0150 & 0 & -0.0572 \\
-0.0172 & 0 & 0.2560 \\
8.9932 \times 10^{-7} & 0 & -7.1218 \times 10^{-7}
\end{array}\right) \\
& K_{2}=\left(\begin{array}{ccc}
35.5841 & 0 & -36.2347 \\
0.1270 & 0 & -2.4444 \\
21.7830 & 0 & -130.0642 \\
8.3810 \times 10^{-7} & 0 & -6.6695 \times 10^{-7} \\
-143.8215 & 0 & 200.2627 \\
-2.1831 & 0 & 10.1451 \\
-116.5421 & 0 & 661.2573 \\
9.3783 \times 10^{-7} & 0 & -6.9896 \times 10^{-7} \\
243.6176 & 0 & -446.0330 \\
7.8905 & 0 & -19.4836 \\
263.4774 & 0 & -1.4117 \times 10^{3} \\
8.4298 \times 10^{-7} & 0 & -6.6690 \times 10^{-7} \\
-193.4364 & 0 & 463.0827 \\
-11.1942 & 0 & 18.5535 \\
-284.7824 & 0 & 1.4271 \times 10^{3} \\
8.5434 \times 10^{-7} & 0 & -6.9896 \times 10^{-7}
\end{array}\right) \\
& K_{4}=\left(\begin{array}{ccc}
K_{3} \\
-190
\end{array}\right)
\end{aligned}
$$




$$
\begin{gathered}
K_{6}=\left(\begin{array}{ccc}
62.1699 & 0 & -184.2103 \\
6.1130 & 0 & -7.2484 \\
121.6514 & 0 & -558.0424 \\
6.7022 \times 10^{-7} & 0 & -5.5183 \times 10^{-7}
\end{array}\right) \\
K_{7}=\left(\begin{array}{ccc}
0.3383 & 0 & 0.1074 \\
2.3482 \times 10^{-3} & 0 & 21.2326 \times 10^{-3} \\
-0.3684 & 0 & 1.1766 \\
8.9820 \times 10^{-7} & 0 & -7.1121 \times 10^{-7}
\end{array}\right)
\end{gathered}
$$

Matrices $K_{j}$ for a fault vector equal to $F=\left[\begin{array}{lll}0 & 1 & 0\end{array}\right]^{T}$ :

$$
\begin{aligned}
K_{1}= & \left(\begin{array}{ccc}
0.6954 & -0.7069 & 0 \\
-1.1278 \times 10^{-3} & 0.2990 & 0 \\
-11.3542 \times 10^{-3} & 0.1764 & 0 \\
-3.1547 \times 10^{-5} & -4.9406 \times 10^{-4} & 0
\end{array}\right) \\
K_{2}= & \left(\begin{array}{ccc}
38.9388 & -58.1434 & 0 \\
-0.1276 & 4.4628 & 0 \\
17.0758 & 82.6757 & 0 \\
6.9342 \times 10^{-5} & 1.0204 \times 10^{-3} & 0
\end{array}\right) \\
K_{3}= & \left(\begin{array}{ccc}
-162.0358 & 319.5950 & 0 \\
-0.9691 & -21.4814 & 0 \\
-91.9862 & -435.4859 & 0 \\
1.9191 \times 10^{-5} & 2.9529 \times 10^{-4} & 0 \\
283.0694 & -697.8027 & 0 \\
5.2561 & 46.9645 & 0 \\
209.3752 & 966.2187 & 0 \\
3.5039 \times 10^{-5} & 5.2213 \times 10^{-4} & 0
\end{array}\right)
\end{aligned}
$$




$$
\begin{aligned}
K_{5} & =\left(\begin{array}{ccc}
-233.2794 & 704.4033 & 0 \\
-8.5758 & -46.6604 & 0 \\
-227.9262 & -1.0137 \times 10^{3} & 0 \\
1.1388 \times 10^{-4} & 1.6783 \times 10^{-3} & 0
\end{array}\right) \\
K_{6} & =\left(\begin{array}{ccc}
77.7121 & -275.0765 & 0 \\
5.0731 & 18.5601 & 0 \\
98.3034 & 416.5143 & 0 \\
9.8984 \times 10^{-5} & 1.4689 \times 10^{-3} & 0
\end{array}\right) \\
K_{7} & =\left(\begin{array}{ccc}
0.3161 & 0.3329 & 0 \\
-12.5148 \times 10^{-3} & 0.2681 & 0 \\
-0.3404 & -0.4086 & 0 \\
3.1325 \times 10^{-5} & 4.6642 \times 10^{-4} & 0
\end{array}\right)
\end{aligned}
$$

\section{References}

R. Isermann, P. Balle, Trends in the application of model based fault detection and diagnosis of technical processes, Control Engineering Practice 5 (1997) 709-719.

M. Rodrigues, D. Theilliol, S. Aberkane, D. Sauter, Fault tolerant control design for polytopic LPV system, International Journal of Applied Mathematics and Computer Science 171 (2007) 27-37.

T. J. Harris, C. Seppala, L. D. Desborough, A review of performance monitoring and assessment techniques for univariate and multivariate control systems., Journal Process of Control 9 (1999) 1-17. 
V. Venkatasubramanian, R. Rengaswamy, K. Yin, S. Kavuri, A review of process fault detection and diagnosis. parti: Quantitative model-based methods, Computers and Chemical Engineering 27 (2003a) 293-311.

V. Venkatasubramanian, R. Rengaswamy, S. Kavuri, A review of process fault detection and diagnosis. partii: Qualitative models-based methods, Computers and Chemical Engineering 27 (2003b) 313-326.

D. Knittel, E. Laroche, D. Gigan, H. Koc, Tension control for winding systems with two degrees of freedom hinfinity controller, IEEE Transactions on Industry Applications 39 (2003) 113-120.

J. Gertler, Fault detection and diagnosis in engineering systems, NYBasel HK: Marcel Dekker,Inc (1998).

J. Chen, R. Patton, Robust model-based fault diagnosis for dynamic systems, Kluwer Academic Publishers (1999).

L. Chiang, E. Russell, R. Braatz, Fault detection and diagnosis in industrial systems, Springer-Verlag, NewYork,USA, 2001.

S. Ding, Model-based fault diagnosis techniques design schemes, algorithms and tools, Springer, 2008.

J. Bokor, Z. Szabo, Fault detection and isolation in nonlinear systems, Annual Reviews in Control. 33 (2009) 113-123.

C. Poussot-Vassal, O. Sename, L. Dugard, P. Gspr, Z. Szab, J. Bokor, A new semi-active suspension control strategy through LPV technique, Control Engineering Practice 16 (2008) 1519-1534. 
J. Bokor, G. Balas, Detection filter design for LPV systemsa geometric approach, Automatica 40 (2004) 511-518.

A. Zolghadri, D. Henry, S. Grenaille, Fault diagnosis for LPV systems, 16th Mediterranean Conference on Control and Automation, MED08, Ajaccio, 2008.

S. Armeni, A. Casavola, E. Mosca, Robust fault detection and isolation for lpv systems under a sensitivity constraint, International Journal of Adaptive Control and Signal Processing 23 (2009) 55-72.

H. Hamdi, M. Rodrigues, C. Mechmeche, D. Theilliol, N. B. Braiek, Fault detection and isolation in linear parameter-varying descriptor systems via proportional integral observer, International Journal of Adaptive Control and Signal Processing 26 (2012) 224-240.

Y. Xiao, S. Huang, H. Chen, B. Xu, Research on tension control strategy of battery pole-piece winding and unwinding system based on fault-tolerant control, Procedia Engineering 15 (2011) 5073-5078.

F. Claveau, P. Chevrel, K. Knittel, A 2dof gain-scheduled controller design methodology for a multi-motor web transport system, Control Engineering Practice 16 (2008) 609-622.

D. Kuhm, D. Knittel, M.-A. Bueno, Robust control strategies for an electric motor driven accumulator with elastic webs, ISA Transactions (2012) In Press, Corrected Proof. 
V. Gassmann, D. Knittel, Robust pi-lpv tension control with elasticity observer for roll-to-roll systems, in: Proceedings of the 18th World Congress, IFAC11, Milano, Italia.

A. Benlatreche, D. Knittel, E. Ostertag, Robust decentralised control strategies for large-scale web handling systems, Control Engineering Practice 16 (2008) 736-750.

D. Theilliol, J.-C. Ponsart, M. Rodrigues, S. Aberkane, J. Yam, Design of sensor fault diagnosis method for nonlinear systems described by linear polynomial matrices formulation: application to a winding machine, Proceedings of the 17th World Congress, IFAC08, Seoul, Korea, 2008.

J.-C. Ponsart, D. Theilliol, C. Aubrun, Virtual sensors design for active fault tolerant control system applied to a winding machine, Control Engineering Practice (2010) 1037-1044.

M. Rodrigues, D. Theilliol, M. Adam-Medina, D. Sauter, A fault detection and isolation sheme for industrial systems based on multiple operationg models, Control Engineering Practice 16 (2008) 225-239.

E. Laroche, D. Knittel, An improved linear fractional model for robustness analysis of a winding system, Control Engineering Practice 13 (2005) 659666.

F. Anstett, G. Millerioux, G. Bloch, Polytopic observer design for LPV systems based on minimal convex polytope finding, Journal of Algorithms and Computational Technology 3 (2009) 23-43. 
F. Bruzelius, Linear Parameter-Varying Systems an approach to gain scheduling, Department of Signals and Systems, Chalmers University of Technology, Goteborg, Sweden, 2004.

J. Park, G. Rizzoni, W. Ribbens, On the representation of sensor faults in fault detection filters, Automatica 30 (1994) 1793-1795.

J. Keller, Fault isolation filter design for linear stochastic systems, Automatica 35 (1999).

P. Frank, X. Ding, Survey of robust residual generation and evaluation methods in observer-based fault detection systems, Journal of Process Control 7 (1997) 403-424.

S. Leonhardt, M. Ayoubi, Methods of fault diagnosis, Control Engineering and Practice 5 (1997) 683-692.

H. Noura, D. Theilliol, J.-C. Ponsart, A. Chamseddine, Fault-Tolerant Control Systems: Design and Practical Applications, Springer, 2009.

L. Hetel, J. Daafouz, C. Iung, Lmi control design for a class of exponential uncertain systems with application to network controlled switched systems, American Control Conference ACC, New York City, USA, CD Rom, 2007.

A. Fossard, D. Normand-Cyrot, Nonlinear systems 1: Modeling and estimation, Chapman and Hall Edition (1995).

A. Isidori, Nonlinear Control Systems, (3e Edition), Springer, 1995. 